\title{
The impact of cold therapy on the incidence and severity of paclitaxel induced peripheral neuropathy: A pilot study
}

\author{
Jawaid Younus*1, Lyn Kligman ${ }^{1}$, Dayam Jawaid ${ }^{2}$ \\ ${ }^{1}$ Medical Oncology, London Regional Cancer Program, London, ON, Canada \\ ${ }^{2}$ Department of Social Sciences, King's College, London, ON, Canada
}

\author{
Received: April 13, 2016 \\ Accepted: May 9, 2016 \\ Online Published: June 5, 2016 \\ DOI: $10.5430 /$ jst.v6n2p43 \\ URL: http://dx.doi.org/10.5430/jst.v6n2p43
}

\begin{abstract}
Background: Peripheral neuropathy is a common side effect induced by chemotherapy agents like Cisplatin, Paclitaxel and Docetaxel. At present, there is no preventive strategy available against the development of neuropathy. This pilot study was performed to evaluate the feasibility of cold therapy and its impact on the incidence and severity of neuropathy induced by paclitaxel in a dose dense adjuvant regimen among patients with breast cancer.

Methods: All adult female patients with early breast cancer planned to be treated with dose-dense schedule of Adriamycin, cyclophosphamide and Paclitaxel were eligible. Ice boot and glove were applied for the duration of all four Paclitaxel infusions on one side while the contralateral limbs were taken as control. Peripheral neuropathy was evaluated and graded prior to each Paclitaxel infusion and then at 3 and 6 months post treatment.

Results: A total of 23 patients were recruited. Cold therapy was found to be feasible as no patient discontinued the ice glove and boot during Paclitaxel infusions. Neuropathy symptoms were observed more frequently and with higher grade of severity on control limbs compared to experimental side. There were 5 patients who were switched to ice glove and boot therapy after they developed peripheral neuropathy in the control limbs. These patients observed improvement in their symptoms subsequently. Conclusion: Cold therapy appears to be feasible and appears to have some potential to prevent Paclitaxel induced peripheral neuropathy in this pilot study. This role of cold therapy may be further explored and confirmed in future randomized trials.
\end{abstract}

Key Words: Hypothermia, Cold therapy, Peripheral neuropathy, Paclitaxel, Breast cancer

\section{INTRODUCTION}

Peripheral neuropathy is seen as a common side effect of many chemotherapy drugs used in both the adjuvant and metastatic disease setting for the treatment of breast cancer. These include cisplatin, docetaxel, and paclitaxel, where the associated distal neuropathy commonly presents with symptoms of burning dysesthesia, numbness, tingling, and shooting pain. ${ }^{[1,2]}$ Such symptoms usually appear first in the feet, but a significant number of patients will report symptoms beginning in the hands. The distribution is predominantly in a symmetrical stocking and glove pattern. ${ }^{[3,4]}$

Demyelination of large sensory nerve fibers as well as axonal degeneration and demyelination has been proposed as the underlying cause of sensory and motor neuropathy subsequent to exposure to these drugs. There is marked variability between patients in the severity and duration of symptoms

*Correspondence: Jawaid Younus; Email: Jawaid.Younus@1hsc.on.ca; Address: 790 Commissioners Road East, London, ON, N6A 4L6, Canada. 
and not all patients will exhibit toxic effects. This nerve injury also appears to be dose related. ${ }^{[5]}$

The incidence of neuropathy may vary with the chemotherapy drug and its dose but reportedly is around 20\%-100\% with paclitaxel. ${ }^{[5-7]}$ The risk of neurotoxicity is increased with the concomitant administration of these neurotoxic drugs such as a combination of cisplatin with paclitaxel or docetaxel.

At present, there are no specific interventions that would prevent the development of peripheral neuropathy. Current management strategy is individual dose reduction or discontinuation of treatment. A number of studies of various agents to treat peripheral neuropathy have met with variable or no success. Most patients will have improvement of symptoms over months to years post treatment with a small number developing chronic, treatment resistant neuropathy. ${ }^{[8,9]}$

There are no published reports on cold therapy as preventive strategy for chemotherapy induced peripheral neuropathy. Anecdotal reports by patients of improvement or disappearance of peripheral neuropathy symptoms with the use of cold therapy used to prevent nail changes with docetaxel, generated the hypothesis for this study. ${ }^{[10]}$ This pilot study was conducted to explore the feasibility of using ice gloves and boots and to observe any impact on the development and severity of peripheral neuropathy symptoms.

\section{HYPOTHESIS}

The application of ice gloves and boots to the hands and feet of patients undergoing infusion of paclitaxel, given every two weeks, on the adriamycin, cyclophosphamide and paclitaxel therapy (AC-P dose dense regimen), is a feasible strategy that may prevent/reduce the incidence/severity (grade) of peripheral neuropathy.

Objectives:

(1) To determine the feasibility of the use of ice gloves and boots during the infusion of paclitaxel chemotherapy.

(2) To observe the incidence and severity of peripheral neuropathy in the ice treated limbs as compared to the non-treated side.

\section{Methods}

The study was approved by the ethics committee of University of Western Ontario. All adult female patients with a confirmed histological diagnosis of breast cancer were eligible to participate, if undergoing adjuvant dose dense AC-P chemotherapy (adriamycin $60 \mathrm{mg} / \mathrm{m}^{2}$, cyclophosphamide $600 \mathrm{mg} / \mathrm{m}^{2}$ IV every two weeks for 4 doses followed by paclitaxel $175 \mathrm{mg} / \mathrm{m}^{2}$ IV once every two weeks for 4 doses) at
London Regional Cancer Program, London, ON. The study was designed as an interrupted time series with multiple replications of the application of an ice glove and boot to one (same side as surgery) hand and foot using the contralateral side as a control, during the infusion of paclitaxel in the chemotherapy suite. This approach reduced the number of patients needed for the study. In addition, the evaluation of a subjective symptom like peripheral neuropathy was considered easier if the same patient could compare the two sides about the incidence and severity of the symptom. Patients with previous exposure to taxanes or with prior diagnosis of peripheral neuropathy from any etiology were excluded. Patients with bilateral breast cancer and those unable to give consent or unable to describe or document symptoms were also excluded.

Participants were identified, reviewed, and consented from the breast disease site clinics of the LRCP. An initial neuropathy screening assessment was done after completion of the four cycles of adriamycin and cyclophosphamide portion of the regimen. A set of standing (repeat) orders were written for the chemotherapy unit nurses to apply an ice glove and boot to the surgical side (right or left hand and foot) for the duration of each paclitaxel infusion. We used Elastogel ${ }^{\circledR}$ gloves and boots (Kansas City, USA). The ice gloves and boots were specified to be replaced every $30 \mathrm{~min}$ utes during the infusion and tolerability was documented by the chemotherapy unit nurses. The chemotherapy nurses have been using these gloves and boots prior to this study and were well trained. These gloves and boots were kept in the freezer according to manufacturer's recommendations prior to their use.

The peripheral neuropathy grade was judged according to NCIC (National Cancer Institute of Canada) common toxicity rating criteria (CTC) as grade 0 through 5 , as described in version 4.03 (http://evs.nci.nih.gov/ftp1/CTCAE/C TCAE_4.03_2010-06-14_QuickReference_5x7.pdf).

Considering this study as primarily pilot and exploratory in nature, no formal statistics were considered in the design and in the interpretation of results. Similarly, NCI-CTC grading was chosen to reflect a simple clinical assessment in the clinic to assess peripheral neuropathy. The assessments were done at five points in time: after completion of four cycles of Adriamycin and Cyclophosphamide; after paclitaxel cycles 1 through 3 and at a 3 months and 6 months follow up visit. The incidence of neuropathy was recorded and graded comparing the treated (iced) side to the untreated (non-iced) side. Each patient was assessed five times for neuropathy on the left side and five times on the right side. Each reported incident was counted once. Patients who developed neuro- 
pathic symptoms on the control limb but not the iced limb were permitted to then begin icing both sides.

\section{RESULT}

A total of thirty three patients were identified as possible participants. Of these, two declined after realizing that they would not be able to freely use their hands for craft or reading activities during the infusion time. Paclitaxel regimen changes from every two weeks to weekly claimed five other possible participants. Three other patients declined for unspecified reasons. Twenty three patients were enrolled to explore the primary and secondary objectives, over a period of 14 months.

The data was complete for 23 patients who participated in this study with the exception of one patient who died between the 3 and 6 months follow up. Considering feasibility, there was no discontinuation of ice glove or boot by any patient due to cold intolerability.
Table 1. Incidence of neuropathy any grade observed in iced (Experiment) versus non-iced (Control) limbs

\begin{tabular}{lll}
\hline Observation & Control limb (n) & Experiment limb (n) \\
\hline Post taxol circle 1 & 6 & 3 \\
Post taxol circle 2 & 9 & 3 \\
Post taxol circle 3 & 13 & 6 \\
At 3 month follow up & 10 & 8 \\
At 6 month follow up & 9 & 7 \\
\hline
\end{tabular}

There was no neuropathy symptoms reported in 67 controls compared to 87 experimental limbs. The neuropathy related problems were observed in 47 controls versus only 27 experimental limbs. The development of any grade neuropathy was evident more on the control side limbs at all time points (see Table 1). The application of ice glove and boot limited the incidence of any grade neuropathy to only 7 patients at 6 months follow up. At 6 months mark, 9 patients on the control side still had symptom of neuropathy (see Table 1).

Table 2. Grades of neuropathy in control and experimental sides

\begin{tabular}{|c|c|c|c|c|c|c|}
\hline \multirow{2}{*}{ Observation } & \multicolumn{3}{|c|}{ Control limb (non-iced) } & \multicolumn{3}{|c|}{ Experiment limb (iced) } \\
\hline & Grade 1 & Grade 2 & Grade 3 & Grade 1 & Grade 2 & Grade 3 \\
\hline Post taxol circle 1 & 2 & 3 & 1 & 3 & & \\
\hline Post taxol circle 2 & 8 & 1 & & 3 & & \\
\hline Post taxol circle 3 & 12 & 1 & & 6 & & \\
\hline At 3 months follow up & 9 & 1 & & 8 & & \\
\hline At 6 months follow up & 7 & 2 & & 7 & & \\
\hline
\end{tabular}

When analyzed for the grade of neuropathy (see Table 2), the experimental arm developed only grade 1 neuropathy at any point in time during the study. In the control group, grade 1 neuropathy was observed most frequently but there was one case of grade 3 neuropathy and several patients reported grade 2 neuropathy.
There were 5 patients who developed neuropathy in the control limb after cycle 1 . These patients were permitted to use ice gloves and boots on both sides. In all such cases, the neuropathy was reduced to a mild grade with the exception of one that persisted through follow up at a moderate level in the control limb (see Table 3).

Table 3. The grades of neuropathy are listed for these 5 patients at various time points for both treated control and experiment side

\begin{tabular}{clllll}
\hline & $\begin{array}{l}\text { Post taxol \#1 } \\
\text { Neuropathy }\end{array}$ & $\begin{array}{l}\text { Post taxol \#2 } \\
\text { Neuropathy }\end{array}$ & $\begin{array}{l}\text { Post taxol \#3 } \\
\text { Neuropathy }\end{array}$ & $\begin{array}{l}\text { Post taxol \#4 } \\
\text { 3 months follow up } \\
\text { Neuropathy }\end{array}$ & $\begin{array}{l}\text { 6 months follow up } \\
\text { Neuropathy }\end{array}$ \\
\hline Pt \#1 control & 2 & 1 & 1 & 0 & 0 \\
experiment & 0 & 0 & 1 & 0 & 0 \\
Pt \#2 control & 3 & 1 & 2 & 0 & 0 \\
experiment & 1 & 1 & 0 & 1 & 0 \\
Pt \#3 control & 1 & 1 & 1 & 0 & 0 \\
experiment & 0 & 0 & 0 & 0 & 0 \\
Pt \#4 control & 1 & 1 & 1 & 1 & 1 \\
experiment & 0 & 0 & 0 & 0 & 0 \\
Pt \#5 control & 2 & 2 & 1 & 1 & 1 \\
experiment & 0 & 1 & 1 & 1 & 1 \\
\hline
\end{tabular}




\section{Discusssion}

We have shown in this pilot study, for the first time, that the use of ice glove and boot may have potential to reduce the incidence and the severity of peripheral neuropathy in patients treated with paclitaxel. This approach appears feasible and reasonably well tolerated by the patients.

The incidence of neuropathy with paclitaxel is $50 \%$ to $100 \%$ which is dose dependent and occurs with higher cumulative dose and higher dose per cycle. ${ }^{[8]}$ A higher incidence of paclitaxel neuropathy has been reported when paclitaxel is infused over three hours as compared to 24 hours. ${ }^{[1]}$ We observed decreased incidence of neuropathy in the limbs treated with ice gloves and boots compared to controls during and post treatment with paclitaxel. In addition, iced limbs did not develop neuropathy more severe than grade 1, compared to non-iced limbs where frequent grade 2 and one grade 3 neuropathy was reported. Interestingly, patients who were switched to ice glove and boot therapy demonstrated impact of cold therapy on the developed neuropathy with improvement over time. We observed pattern of neuropathy similar to the previously published studies. In this study, fingertips and toes were the first to be affected and the feet were the last to resolve. Only two of the twenty three subjects had ongoing moderate neuropathy at 6 months but this was only on the non-iced side. One patient had onset of moderate neuropathy 3 months post completion on the non-iced side and the iced side rated as mild.

There are no studies in published literature, at present, exploring the impact of hypothermia to prevent peripheral neuropathy in patients treated with chemotherapy. The exact mechanism by which a paclitaxel induced peripheral neuropathy could get potentially impacted by hypothermia is not known. However, the cooling techniques in a variety of local appli- cation forms have been used to prevent or minimize other conditions like alopecia resulting from chemotherapy. ${ }^{[12-14]}$ The hypotheses behind the impact of hypothermia for a localized area have included a variety of proposed factors, ranging from decreased metabolic activity of cells in the local area to decreased blood flow resulting in less drug exposure.

There are several weaknesses in this pilot study. This study is primarily observational, without formal statistics and limited to only 23 patients. We did not include demographics and details about type of surgery about these patients. We felt that cold therapy is likely unaffected by these parameters as a strategy against peripheral neuropathy. Although surgery induced neuropathy is possible, we excluded patients experiencing neuropathy from any etiology. In addition, we applied the cold therapy on the surgery side only. Permitting patients to ice both hands and feet was felt to be ethically responsible, but did result in loss of information as to the perhaps eventual severity of neuropathy on the non-iced side but did show that the application of ice glove and boot reduced the severity in subsequent cycles. The three hour infusion of paclitaxel was a long time to have cold treatment and patients who did report onset of neuropathy on the iced side frequently admitted that they had engaged in cold avoidant behaviors such as curling up their fingers inside the ice mitt or wearing socks inside the ice boot.

Despite these practical issues, the improved incidence and grade of neuropathy observed with ice glove and boot application are promising and provide a potential option of preventive strategy that should be explored through randomized clinical trials in future.

\section{CONFlicts OF INTEREST Disclosure}

The authors declare that there is no conflict of interest statement.

\section{REFERENCES}

[1] Dougherty P, Cata J, Cordella J, et al. Taxol-induced sensory disturbance is characterized by preferential impairment of myelinated fiber function in cancer patients. Pain. 2004; 109: 132-42. PMid:15082135. http://dx.doi.org/10.1016/j.pain.2004.01.021

[2] Argyriou A, Koltzenburg M, Polychronopoulos P, et al. Peripheral nerve damage associated with administration of taxanes in patients with cancer. Critical Reviews in Oncology/Hematology. 2008; 66: 218-28. PMid:18329278. http://dx.doi.org/10.1016/j.cri trevonc. 2008.01.008

[3] Scripture C, Figg W, Sparreboom A. Peripheral neuropathy induced by paclitaxel: recent insights and future perspectives. Current Neuropharmacology. 2006; 4: 165-72. PMid:18615126. http: //dx.doi.org/10.2174/157015906776359568
[4] Kuroi K, Shimozuma K. Neurotoxicity of taxanes: symptoms and quality of life assessment. Breast Cancer. 2004; 11 (1): 92-9. PMid:14718800. http://dx.doi.org/10.1007/BF02968010

[5] Park S, Lin C, Krishnan A, et al. Early, progressive, and sustained dysfunction of sensory axons underlies paclitaxel-induced neuropathy. Muscle Nerve. 2011; 43 (3): 367-74. PMid:21321953. http://dx.doi.org/10.1002/mus. 21874

[6] Reyes-Gibby C, Morrow P, Buzdar A, et al. Chemotherapy -induced peripheral neuropathy as a predictor of neuropathic pain in breast cancer patients previously treated with paclitaxel. Journal Pain. 2009; 10 (11): 1146-50. PMid:19595634. http://dx.doi.org/10.10 $16 / j \cdot j$ pain. 2009.04.006

[7] Pace A, Nistico C, Cuppone F, et al. Peripheral neurotoxicity of weekly paclitaxel chemotherapy: a schedule or a dose issue? Clin 
Breast Cancer. 2007 Apr; 7 (7): 550-4. PMid:17509163. http: //dx.doi.org/10.3816/CBC.2007.n.010

[8] Park S, Goldstein D, Krishnan A, et al. Chemotherapy-induced peripheral neurotoxicity: A critical analysis. CA: A Cancer Journal for Clinicians. 2013; 63(6): 419-37. http://dx.doi.org/10.3322

/caac. 21204

[9] Kannarkat G, Lasher E, Shiff D. Neurologic complications of chemotherapy agents. Current Opinion in Neurology. 2007; 20: 71925. PMid:17992096.

[10] Gilbar P, Hain A, Peereboom V. Nail toxicity induced by cancer chemotherapy. Journal Oncology Pharm Practice. 2009; 15: 14355. PMid:19171552. http://dx.doi.org/10.1177/107815520 8100450
[11] Cleeland C, Farrar J, Hausheer F. Assessment of cancer -related neuropathy and neuropathic pain. The Oncologist. 2010; 15(suppl 2): 13-8. PMid:20489192. http://dx.doi.org/10.1634/theon cologist.2009-S501

[12] Satterwhite B, Zimm S. The use of scallop hypothermia in the prevention of doxorubicin-induced hair loss. Cancer. 1984: 54(1): 34. http://dx.doi.org/10.1002/1097-0142(19840701) 54: $1<34$ : : AID-CNCR2820540109>3.0.CO;2-W

[13] Macduff C, Mackenzie T, Hutcheon A, et al. The effectiveness of scalp cooling in preventing alopecia for patients receiving epirubicin and docetaxel. Eur J Cancer Care (Engl). 2003 Jun; 12(2): 154-61. http://dx.doi.org/10.1046/j.1365-2354.2003.00382.x

[14] Parker R. The effectiveness of scalp hypothermia in preventing cyclophosphamide-induced alopecia. Oncol Nurs Form. 1987; 14(6): 49. PMid:3697290. 